\title{
On the Random Sampling Amplitude Error
}

\author{
Shouyuan Yang ${ }^{1}$, Zhanjie Song ${ }^{2, \star}$, and Xingwei Zhou ${ }^{3, \star \star}$ \\ ${ }^{1}$ Department of Mathematics, Naikai University and LPMC, \\ Tianjin 300071, China \\ yshouy@sina.com \\ 2 School of Science, Tianjin University, \\ Tianjin 300072, China \\ zhanjiesong@tju.edu.cn \\ ${ }^{3}$ Department of Mathematics, Naikai University and LPMC, \\ Tianjin 300071, China \\ xwzhou@naikai.edu.cn
}

\begin{abstract}
The main purpose of this paper is to examine the distribution of the random amplitude error for the sampling problem in diverse situations, and specific formulas are given, which reveal the connection between the random errors of the sampled values and the amplitude error caused by them. The information loss error is also included as a special case.
\end{abstract}

\section{Introduction and Preliminaries}

Sampling theories are now widely used in many areas, especially in digital signal processing and transmitting. The most important feature of all sampling theorems is that a continuous signal can be recovered from a sequence of sampled values. The most famous sampling theorem which is usually attributed to Shannon stated that

$$
f(t)=\sum_{n \in Z Z} f\left(\frac{n}{2 \sigma}\right) \frac{\sin 2 \pi \sigma(t-n / 2 \sigma)}{2 \pi \sigma(t-n / 2 \sigma)}
$$

for any $\sigma$-bandlimited signals $f(t)$, i.e., $f(t) \in L^{2}(\mathbb{R})$ and its Fourier transform $\hat{f}(\xi):=\int_{\mathbb{R}} f(t) e^{-i 2 \pi \xi t} d t$ supported on $[-\sigma,+\sigma]$, where $L^{2}(\mathbb{R})$ denote the space of all square integrable signals. The classical sampling theorem has been extended in many ways during the last five decades. The most important extension may be nonuniform sampling and sampling in other signal spaces, such as splinelike (shift-invariant) spaces and wavelet subspaces, e.g., see [1, 4, 5, 10, Higher dimensional sampling is also considered by many researchers because of its wide application in image processing and many other areas.

\footnotetext{
* Supported by the National Natural Science Foundation of China (60476042 and 60572113), the Liuhui Center for Applied Mathematics.

** Supported by the Natural Science Foundation of China under Grant 60472042 and the Research Fund for the Doctoral Program of Higher Education.
} 
Now let us introduce some notations. We use $\mathbb{R}^{d}$ and $Z^{d}$ to denote the $d$ dimensional Euclidean space and unit lattice, respectively. $L\left(\mathbb{R}^{d}\right)$ and $l^{2}\left(Z^{d}\right)$ denote the space of all square integrable signals defined on $\mathbb{R}^{d}$ and the space of all square summable sequences defined on $Z^{d}$, respectively. With the inner product $\langle f, g\rangle=\int_{\mathbb{R}^{d}} f(t) \overline{g(t)} d t, L^{2}\left(\mathbb{R}^{d}\right)$ constitutes a Hilbert space. Obviously, the sampling problem would be meaningless if no restriction is imposed on the signal space and the set of sampling points. Throughout this paper we assume that the signal space $V \subseteq L^{2}\left(\mathbb{R}^{d}\right)$ and the set of sampling points $X:=\left\{t_{j}\right\}_{j \in J} \subseteq$ $\mathbb{R}^{d}$ satisfy the following conditions:

i). There exists a sequence $\left\{s_{n}: n \in \mathbb{Z}^{d}\right\}$ of functions in $V$ which is called a sampling sequence of $V$ such that

$$
f(t)=\sum_{n \in Z Z^{d}} f\left(t_{n}\right) s_{n}(t)
$$

for any $f \in V$, where the convergence is in the $L^{2}\left(\mathbb{R}^{d}\right)$-sense. In particular, if there exist $s \in V$ such that $\left\{s\left(\cdot-t_{n}\right): n \in \mathbb{Z}^{d}\right\}$ constitutes a sampling sequence of $V$, then $s$ is said to be a sampling function.

ii). The sampling operator $\mathrm{S}_{X}: V \rightarrow l^{2}(X)$ defined by $\mathrm{S}_{X} f=\left(f\left(t_{j}\right)\right)_{j \in J}$ is a bounded linear operator, i.e.,

$$
\sum_{j \in J}\left|f\left(t_{j}\right)\right|^{2} \leq B\|f\|_{2}^{2}, \text { for all } f \in V,
$$

where $B$ is a constant independent of $f$.

It is worthwhile pointing out that so far all the sampling theorems either include the above conditions as a assumption or include other assumptions from which the above conditions can be obtained as a conclusion. Here we list the sampling sequence or sampling functions for several well-known sampling problems:

I) Uniform sampling for band-limited functions. The signal space is $B_{\sigma}$, which consists of all $\sigma$-bandlimited signals defined on $\mathbb{R}$, the system $\{\operatorname{sinc} 2 \pi \sigma(\cdot-$ $n / 2 \sigma)\}_{n \in Z Z}$ constitutes a sampling sequence of $B_{\sigma}$, where sinc $t:=\sin t / t$. Hence sinc $2 \pi \sigma(\cdot)$ is a sampling function of $B_{\sigma}$, the reconstruction formula is exactly the Shannon sampling theorem.

II) If $\sigma=1,\left\{t_{n}\right\}_{n \in Z Z}$ is a sequence of real numbers such that $\left|t_{n}-n\right| \leq$ $L<1 / 4$ for all $n$, then by Kadec's $\frac{1}{4}$-theorem (e.g., see [1]), the sequence $\left\{G_{n}(t)\right\}_{n \in Z Z}$ constitutes a sampling sequence of $B_{\sigma}$, where

$$
G_{n}(t):=\frac{G(t)}{G^{\prime}\left(t_{n}\right)\left(t-t_{n}\right)}, \quad G(t):=t \prod_{n \in Z Z}\left(1-\frac{t^{2}}{t_{n}^{2}}\right) .
$$

III) If the signal space is a spline-like space $V^{2}(\varphi)$ defined as follows

$$
V^{2}(\varphi):=\left\{\sum_{n \in Z^{d}} c_{n} \varphi(\cdot-n): c=\left(c_{n}\right) \in l^{2}\left(\mathbb{Z}^{d}\right)\right\},
$$


where $\varphi$ satisfies

$$
0<c \leq G_{\varphi}(\xi)=\sum_{j \in Z^{d}}|\hat{\varphi}(\xi+j)|^{2} \leq C, \text { a.e. } \xi \in \mathbb{R}^{d}
$$

and some decay and smoothness condition, e.g., $\varphi$ is continuous and satisfies

$$
\|\varphi\|_{W\left(L^{p}\left(\mathbb{R}^{d}\right)\right)}:=\left(\sum_{k \in Z^{d}} \sup _{t \in[0,1]^{d}}|\varphi(t+k)|^{p}\right)^{1 / p}<\infty,
$$

then the function $s$ determined by

$$
\hat{s}(\xi)=\frac{\hat{\varphi}(\xi)}{\sum_{j \in Z^{d}} \varphi(j) e^{2 \pi i j \cdot \xi}}
$$

is a sampling function of $V^{2}(\varphi)$, and $\{s(\cdot-n)\}_{n \in Z Z^{d}}$ is a sampling sequence, e.g., see [1,10. If the sampling points are not uniformly distributed, we can also construct a sampling sequence of $V^{2}(\varphi)$. Indeed, if we let $\tilde{\varphi}$ be determined by $\hat{\tilde{\varphi}}(\xi)=\hat{\varphi}(\xi) / G_{\varphi}(\xi)$, where $G_{\varphi}(\xi)$ defined in (2), then $K(x, y):=\sum_{j \in Z Z^{d}} \overline{\varphi(x-j)} \tilde{\varphi}(y-j)$ is a reproducing kernel (e.g., see [16]), namely,

$$
f(t)=\langle f, K(t, \cdot)\rangle, \text { for all } t \in \mathbb{R}^{d}, f \in V^{2}(\varphi) .
$$

If the sampling points $\left\{t_{j}\right\}$ are dense enough, then $\left\{K\left(t_{j}, \cdot\right)\right\}$ constitutes a frame for $V^{2}(\varphi)$, and its dual frame $\left\{\widetilde{K\left(t_{j}, \cdot\right)}\right\}$ is what we try to find, e.g., see 1, 13, 14, 15.

IV) Let $\varphi$ be a scaling function (e.g., see [5, 8, 9]) satisfying (2) and certain decay and smoothness condition, $\left\{V_{m}: m \in \mathbb{Z}\right\}$ be the multi-resolution analysis generated by $\varphi$ (e.g., see [5]). If $s$ be the function determined by (3), then for each $m$ the system $\left\{s_{m, n}: n \in Z Z\right\}$ constitutes a sampling basis of $V_{m}$, where $s_{m, n}=\varphi\left(2^{m} \cdot-n\right)$. The reconstruction formula is

$$
f(t)=\sum_{n \in Z^{d}} f\left(\frac{n}{2^{m}}\right) s_{m, n}(t), \text { for all } f \in V_{m} .
$$

There are several type of errors which occur in in the real application of sampling theorems, e.g., see [6]. In 3], Atreas et al examined the truncation error of the reconstruction formula in wavelet subspaces. It was not long before Yang et al extended their results to higher dimensional cases and spline-like spaces, e.g., see [12,14. In this paper we shall investigate the random amplitude error for the above sampling expansions. Specifically, let $f\left(t_{j}\right)$ be the true value of the signal $f$ at the sample $t_{j}$, and $\widetilde{f\left(t_{j}\right)}$ be the sampled value obtained by apparatus, of course it cannot be absolutely precise, since it is often noised by a random error. Let $\lambda\left(t_{j}\right)$ be the relative error defined by

$$
\lambda\left(t_{j}\right):=\frac{\widetilde{f\left(t_{j}\right)}-f\left(t_{j}\right)}{f\left(t_{j}\right)}
$$


if $f\left(t_{j}\right) \neq 0$, otherwise $\lambda\left(t_{j}\right)=\operatorname{sgn}\left(\widetilde{f\left(t_{j}\right)}-f\left(t_{j}\right)\right) \cdot \infty$, where $\operatorname{sgn}(\cdot)$ denotes the sign function, i.e., $\operatorname{sgn}(x)=1$ if $x>0, \operatorname{sgn}(x)=-1$ if $x<0$, and $\operatorname{sgn}(0)=0$, and $0 \cdot \infty=0$ in the definition of $\lambda\left(t_{j}\right)$ by convention. Since the relative error is determined by the inertia of the sampling apparatus and many other unknown factors, it is impossible to find out its precise value, so we assume that all $\lambda\left(t_{j}\right)$ 's are independent and identically distributed (i.i.d.) random variables with finite first moments. The amplitude error is defined by

$$
\operatorname{Am} f(t):=\operatorname{Rec} f\left(t ; \cdots, \widetilde{f\left(t_{j}\right)}, \cdots\right)-f(t),
$$

where $\operatorname{Rec} f\left(t ; \cdots, \widetilde{f\left(t_{j}\right)}, \cdots\right)$ denotes the signal reconstructed from the sequence $\left\{\widetilde{f\left(t_{j}\right)}\right\}$ of measured samples.

\section{Random Amplitude Error Estimation}

In this section we assume that the $L^{2}\left(\mathbb{R}^{d}\right)$-norm of the original signal $f(t)$ is finite, and then examine the distribution of the amplitude error in terms of this norm. We assume henceforth that the relative errors $\lambda\left(t_{j}\right)$ are i.i.d. random variables with $\mathrm{E}\left[\lambda\left(t_{j}\right)\right]=0$ and $\mathrm{E}\left[\left|\lambda\left(t_{j}\right)\right|\right]=\delta<\infty$ if no other assumptions are claimed, where $\mathrm{E}[X]$ denotes the expectation (mean) of the random variable $X$.

\subsection{Uniform Sampling}

Without loss of generality, we assume that the unit lattice $Z^{d}$ and the signal space $V \subseteq L^{2}\left(\mathbb{R}^{d}\right)$ satisfy the conditions i) and ii) given in Section 1 . Let $\{s(\cdot-$ $j): j \in \bar{Z}^{d}$ \} be a sampling sequence of the signal space $V$. Then we have the following reconstruction formula

$$
f(t)=\sum_{j \in Z Z^{d}} f(j) s(t-j)
$$

and the amplitude error can be rewrite as

$$
\begin{aligned}
\operatorname{Am} f(t) & =\operatorname{Rec} f\left(t ; \cdots, \widetilde{f\left(t_{j}\right)}, \cdots\right)-f(t) \\
& =\sum_{j \in Z^{d}} \widetilde{f(j)} s(t-j)-\sum_{j \in Z^{d}} f(j) s(t-j) \\
& =\sum_{j \in Z^{d}} \lambda(j) \cdot f(j) s(t-j) .
\end{aligned}
$$

Hence we have

$$
\mathrm{E}[\operatorname{Am} f(t)]=\sum_{j \in Z^{d}} \mathrm{E}[\lambda(j)] \cdot f(j) s(t-j)=0
$$


and

$$
\begin{aligned}
\mathrm{E}[|\operatorname{Am} f(t)|] & \leq \mathrm{E}\left[\sum_{j \in Z^{d}}|\lambda(j)| \cdot|f(j) s(t-j)|\right] \\
& =\delta \sum_{j \in Z^{d}}|f(j) s(t-j)| \\
& \leq \delta\left(\sum_{j \in Z^{d}}|f(j)|^{2}\right)^{1 / 2} \cdot\left(\sum_{j \in Z^{d}}|s(t-j)|^{2}\right)^{1 / 2} \\
& \leq \delta \cdot B^{1 / 2} \cdot\|f\|_{2} \cdot\left(\sum_{j \in Z^{d}}|s(t-j)|^{2}\right)^{1 / 2} .
\end{aligned}
$$

Now by the Chebyshev's inequality, from (77) and (8) we get that

$$
\operatorname{Prob}\{|\operatorname{Am} f(t)|<\epsilon\} \geq 1-\frac{\delta \cdot B^{1 / 2}}{\epsilon}\|f\|_{2} \cdot\left(\sum_{j \in Z^{d}}|s(t-j)|^{2}\right)^{1 / 2} .
$$

Hence we have proved the first part of the following theorem.

Theorem 1. Let the set of sampling points $\mathbb{Z}^{d}$ and the signal space $V$ satisfy the conditions given in Section 1, and assume that there exists a sampling function $s \in V$ such that the reconstruction formula (5) holds for all $t$. If $s$ decays fast enough such that $\sum_{j \in Z^{d}}|s(t-j)|^{2}<\infty$, then the amplitude error satisfies (9). In particular, if $|s(t)| \leq C\left(1+|t|^{\alpha}\right)^{-1 / 2}$, where $\alpha>d$, then we have

$$
\operatorname{Prob}\{|\operatorname{Am} f(t)|<\epsilon\} \geq 1-\frac{C \cdot \delta \cdot B^{1 / 2} \cdot \alpha^{1 / 2} \cdot 2^{(\alpha+d) / 2}}{\epsilon \cdot(\alpha-d)^{1 / 2}}\|f\|_{2}
$$

for all $t$.

Proof. Only inequality (10) needs to prove. For each $j \in \mathbb{Z}^{d}$, let $Q_{j}$ be a closed ball centred at $j$ with radius $1 / 2$. For $y \in Q_{j}$ and $t \in Q_{j}^{c}$, where $Q_{j}^{c}$ denotes the complement of $Q_{j}$, direct calculations show that

$$
\begin{aligned}
\frac{1+|t-y|^{\alpha}}{1+|t-j|^{\alpha}} & \leq \frac{1+(|t-j|+|y-j|)^{\alpha}}{1+|t-j|^{\alpha}} \\
& \leq \frac{1+(|t-j|+1 / 2)^{\alpha}}{1+|t-j|^{\alpha}} \\
& \leq \frac{1+|t-j|^{\alpha}\left(1+\frac{1}{2|t-j|}\right)^{\alpha}}{1+|t-j|^{\alpha}} \\
& \leq 2^{\alpha} .
\end{aligned}
$$


For $t \in Q_{j}$, we can also prove that $\left(1+|t-y|^{\alpha}\right) /\left(1+|t-j|^{\alpha}\right) \leq 2^{\alpha}$. Hence $\left(1+|t-j|^{\alpha}\right)^{-1} \leq 2^{\alpha} \cdot\left(1+|t-y|^{\alpha}\right)^{-1}$ for all $y \in Q_{j}$ and all $t \in \mathbb{R}^{d}$, and therefore

$$
\begin{aligned}
\sum_{j \in Z^{d}}|s(t-j)|^{2} & \leq C^{2} \sum_{j \in Z^{d}}\left(1+|t-j|^{\alpha}\right)^{-1} \\
& \leq C^{2} \cdot 2^{\alpha} \sum_{j \in Z^{d}}\left|Q_{j}\right|^{-1} \int_{Q_{j}}\left(1+|t-y|^{\alpha}\right)^{-1} d y \\
& \leq C^{2} \cdot 2^{\alpha+d} \frac{d}{S_{d}} \int_{\mathbb{R}^{d}}\left(1+|t-y|^{\alpha}\right)^{-1} d y \\
& \leq C^{2} \cdot 2^{\alpha+d} \cdot d \cdot\left(\frac{1}{d}+\frac{1}{\alpha-d}\right)
\end{aligned}
$$

where $\left|Q_{j}\right|$ and $S_{d}$ denote the volume of the closed ball $Q_{j}$ and the area of the $d$-dimensional unit sphere, respectively. The inequalities (9) and (11) lead to the conclusion immediately.

Note that for band-limited sampling theorems the sampling function can be obtained by dilating the function $\operatorname{sinc}(\cdot)$, therefore, obviously satisfies the decay condition required in Theorem 1 for sampling theorems in the spline-like spaces, the decay of the sampling function is guaranteed by the decay of the generator $\varphi$. Indeed, Yang has proved that in the spline-like spaces the asymptotic rate of decay of the sampling function is the same as that of the generator (see [12]). As for the wavelet subspaces, it can be viewed as a spline-like space generated by the dilated scaling function, so the amplitude error estimate obtained in spline-like spaces can be easily extended to wavelet subspaces.

\subsection{Nonuniform Sampling}

Now let us consider the general case. Let the signal space $V \subseteq L^{2}\left(\mathbb{R}^{d}\right)$ and the set of sampling points $\left\{t_{j}: j \in J\right\}$ satisfy the conditions i) and ii) given in Section 1 . Then the amplitude error can be rewrite as

$$
\operatorname{Am} f(t)=\sum_{j \in J} \lambda\left(t_{j}\right) \cdot f\left(t_{j}\right) s\left(t-t_{j}\right)
$$

By the same techniques we can prove the following results.

Theorem 2. Let the set of sampling points $\left\{t_{j}: j \in J\right\}$ and the signal space $V$ satisfy the conditions given in Section 1. If the sampling sequence $\left\{s_{j} \in V: j \in\right.$ $J\}$ satisfies that $\sum_{j \in J}\left|s_{j}(t)\right|^{2}<\infty$ uniformly, then we have

$$
\operatorname{Prob}\{|\operatorname{Am} f(t)|<\epsilon\} \geq 1-\frac{\delta \cdot B^{1 / 2}}{\epsilon}\|f\|_{2} \cdot\left(\sum_{j \in J}\left|s_{j}(t)\right|^{2}\right)^{1 / 2}
$$


for all $t$. In particular, if $\left|s_{j}(t)\right| \leq C\left(1+\left|t-t_{j}\right|^{\alpha}\right)^{-1 / 2}$ for all $j \in J$, where $\alpha>d$, and the set of sampling points $\left\{t_{j}: j \in J\right\}$ are separated, i.e., $\inf _{j, l \in J, j \neq l} \mid x_{j}-$ $x_{l} \mid=\mu>0$, then we have

$$
\operatorname{Prob}\{|\operatorname{Am} f(t)|<\epsilon\} \geq 1-\frac{C \cdot \delta \cdot B^{1 / 2} \cdot \alpha^{1 / 2} \cdot 2^{(\alpha+d) / 2}}{\epsilon \cdot \mu^{d / 2} \cdot(\alpha-d)^{1 / 2}}\|f\|_{2}
$$

for all $t$.

We point out that for nonuniform sampling in spline-like spaces, the decay of the sampling sequence $\left\{s_{j}: j \in J\right\}$ is also guaranteed by the decay of the generator $\varphi$, e.g., see [7]. Secondly, the constant $B$ appearing in condition ii) in Section 1 depends on the density of the samples, and its existence is guaranteed by the separateness of the samples.

\section{Random Information Loss Error Estimation}

If the relative errors are binary valued, namely, $\lambda\left(t_{j}\right)$ either takes the value 1 or takes the value 0 , no other value is allowed, then the corresponding amplitude error is called the information loss error in [2]. In that paper, the error caused by the missing of some sampled data are considered, where $\lambda\left(t_{j}\right)=1$ for the sampling points $t_{j}$ at which the sampled values $f\left(t_{j}\right)$ are missing and $\lambda\left(t_{j}\right)=0$ otherwise. In the present paper we assume that the missing occurs randomly, and $\lambda\left(t_{j}\right)$ are i.i.d. random variables with $\operatorname{Prob}\left\{\lambda\left(t_{j}\right)=1\right\}=p$ and $\operatorname{Prob}\left\{\lambda\left(t_{j}\right)=\right.$ $0\}=1-p$. Since the following results are just special cases of Theorem 2 , so we omit its proof.

Theorem 3. Let the set of sampling points $\left\{t_{j}: j \in J\right\}$ and the signal space $V$ satisfy the conditions given in Section 1, and $\lambda\left(t_{j}\right)$ be the corresponding relative errors with all the properties stated above. If the sampling sequence $\left\{s_{j} \in V\right.$ : $j \in J\}$ satisfies that $\sum_{j \in J}\left|s_{j}(t)\right|^{2}<\infty$ uniformly, then we have

$$
\operatorname{Prob}\{|\operatorname{Am} f(t)-p|<\epsilon\} \geq 1-\frac{p \cdot B^{1 / 2}}{\epsilon}\|f\|_{2} \cdot\left(\sum_{j \in J}\left|s_{j}(t)\right|^{2}\right)^{1 / 2}
$$

for all $t$. In particular, if $\left|s_{j}(t)\right| \leq C\left(1+\left|t-t_{j}\right|^{\alpha}\right)^{-1 / 2}$ for all $j \in J$, where $\alpha>d$, and the set of sampling points $\left\{t_{j}: j \in J\right\}$ are separated, i.e., $\inf _{j, l \in J, j \neq l} \mid x_{j}-$ $x_{l} \mid=\mu>0$, then we have

$$
\operatorname{Prob}\{|\operatorname{Am} f(t)-p|<\epsilon\} \geq 1-\frac{C \cdot p \cdot B^{1 / 2} \cdot \alpha^{1 / 2} \cdot 2^{(\alpha+d) / 2}}{\epsilon \cdot \mu^{d / 2} \cdot(\alpha-d)^{1 / 2}}\|f\|_{2}
$$

for all $t$. 


\section{References}

1. A. Aldroubi, and K. Gröchenig, Nonuniform sampling and reconstruction in shiftinvariant spaces. SIAM Rev. 43(4)(2001) 585-620

2. N.treas, N.Bagis and C.Karanikas, The information loss error and the jitter error for regular sampling expansions, Sampling Theory in Signal and Image Processing, $\mathbf{1}(3)(2003)$ 261-276

3. N. Atreas, J. J. Benedetto, and C. Karanikas, Local sampling for regular wavelet and Gabor expansions(to appear)

4. John J. Benedetto, Irregular sampling and frames, in: C. K. Chui (Ed.), Wavelets: A Tutorial in Theory and Applications. (1992) 445-507

5. I. Daubechies, Ten Lectures on Wavelets. CBMS-NSF Series in Applied Math. SIAM Philadelphia. 1992.

6. H.Feichtinger and K.Gröchenig, Error analysis in regular and irregular sampling theory, Appl. Anal. 50 (1993) 167-189.

7. K. Gröchenig, Localization of frames, Banach frames, and the invertibility of the frame operator. J. Fourier Anal. Appl.(to appear)

8. S. Mallat, A Wavelet Tour of Signal Processing. Academic Press, Boston, 1998

9. Y. Meyer, Ondelettes Et Opérateurs. Hermann, Paris, 1990

10. Michael Unser, Sampling-50 years after Shannon. Procdings of the IEE. 88 (4) (2000) 569-587

11. R.M.Yang, An Introduction to Nonharmonic Analysis, Academic Press, New York.

12. S. Y. Yang, Local error estimation for samling problems, Appl. Math. Comp. 158 (2004) 561-572.

13. S.Y.Yang, Wavelet Frames, Local Sampling Problems and Applications, PhD thesis, 2004.

14. S.Y.Yang and W.Lin, Local sampling problems, Lecture Notes in Computer Science, 3037 (2004) 81-88.

15. S.Y.Yang, The local property of several operators on sampling, Applicable anal. 83 (9) (2004) 905-913.

16. K. Yao, Application of reproducing kernel Hilbert spaces-bandlimited signal models. Inform. and control. 11 (1967) 429-444 